\title{
Low-grade glioneuronal tumors with FGFR2 fusion resolve into a single epigenetic group corresponding to 'Polymorphous low-grade neuroepithelial tumor of the young'
}

\author{
Rohit Gupta $^{1}$ - Calixto-Hope G. Lucas ${ }^{1} \cdot$ Jasper Wu ${ }^{1}$ · Jairo Barreto ${ }^{1} \cdot$ Kathan Shah ${ }^{1} \cdot$ Iraide Bernal Simon $^{2}$. \\ Sandro Casavilca-Zambrano ${ }^{3}$. Carole Brathwaite ${ }^{4}$. Holly Zhou ${ }^{5}$. Dario Caccamo ${ }^{6}$ - Ahmed Gilani ${ }^{7}$. \\ Bette K. Kleinschmidt-DeMasters ${ }^{7}$. Julieann C. Lee ${ }^{1}$. Arie Perry ${ }^{1,8}$ • Jennifer L. Clarke ${ }^{9,10} \cdot$ Susan M. Chang $^{9}$. \\ Mitchel S. Berger ${ }^{8}$. David A. Solomon ${ }^{1}$ (D)
}

Received: 2 June 2021 / Revised: 20 July 2021 / Accepted: 21 July 2021 / Published online: 28 July 2021

(c) The Author(s) 2021

Low-grade neuroepithelial tumors (LGNET) are a diverse group of neoplasms occurring most commonly in children and young adults, often associated with epilepsy and favorable clinical outcomes. They are composed of a spectrum of tumor entities with divergent clinicopathologic features including ganglioglioma, pilocytic astrocytoma, dysembryoplastic neuroepithelial tumor (DNT), rosette-forming glioneuronal tumor (RGNT), extraventricular neurocytoma (EVN), multinodular and vacuolating neuronal tumor (MVNT), polymorphous low-grade neuroepithelial tumor of

David A. Solomon

david.solomon@ucsf.edu

1 Department of Pathology, University of California, San Francisco, 513 Parnassus Ave, Health Sciences West 451, San Francisco, CA 94143, USA

2 Department of Pathology, University Hospital Donostia, San Sebastián, Spain

3 Department of Pathology, National Institute of Neoplastic Diseases, Lima, Peru

4 Department of Pathology, Nicklaus Children's Hospital, Miami, FL, USA

5 Department of Pathology, University of Utah School of Medicine, Salt Lake City, UT, USA

6 Department of Pathology, Sutter Medical Center, Sacramento, CA, USA

7 Department of Pathology, University of Colorado, Aurora, CO, USA

8 Department of Neurological Surgery, University of California, San Francisco, CA, USA

9 Division of Neuro-Oncology, Department of Neurological Surgery, University of California, San Francisco, CA, USA

10 Department of Neurology, University of California, San Francisco, CA, USA the young (PLTNY), myxoid glioneuronal tumor (MGNT), diffuse leptomeningeal glioneuronal tumor (DLGNT), and papillary glioneuronal tumor (PGNT). However, histologically distinguishing between these different LGNET subtypes can be challenging, and molecular profiling is now recognized as critical for accurate classification. While some LGNET subtypes are defined by unique genetic alterations (e.g. PRKCA fusion in PGNT [4], PDGFRA p.K385L/I dinucleotide mutation in MGNT [9]) that can be used for definitive subtyping, other alterations such as $B R A F$ mutation or fusion are nonspecific and can be seen in ganglioglioma, pilocytic astrocytoma, MVNT, and DLGNT [3, 10-12, 14]. FGFRl is another promiscuous oncogene in LGNET with kinase domain tandem duplication, gene fusions (most often with $T A C C l$ as the fusion partner), or hotspot missense mutations at one of two codons within the tyrosine kinase domain (p.N546 or p.K656) recurrently found in pilocytic astrocytoma, DNT, RGNT, and EVN [8, 12-17, 20]. Thus, additional ancillary methodologies such as DNA methylation profiling may be necessary for accurate classification of LGNET with either $B R A F$ or FGFRI alterations.

Fusions involving the related $F G F R 2$ oncogene have recently been described as one of the characteristic genetic alterations in the newly recognized tumor entity PLNTY, an epileptogenic neoplasm predominantly occurring in the cerebral hemispheres of children and young adults with oligodendroglioma-like components, abundant calcification, and aberrant CD34 expression [5]. However, rare cases of histologically-defined ganglioglioma, MVNT, DNT, oligodendroglioma, and unclassifiable low-grade glioneuronal tumors have also been reported with FGFR2 fusions [6, 10, 11, 14]. To improve classification for such FGFR2-fused tumors, we performed targeted next-generation sequencing and genome-wide DNA methylation 


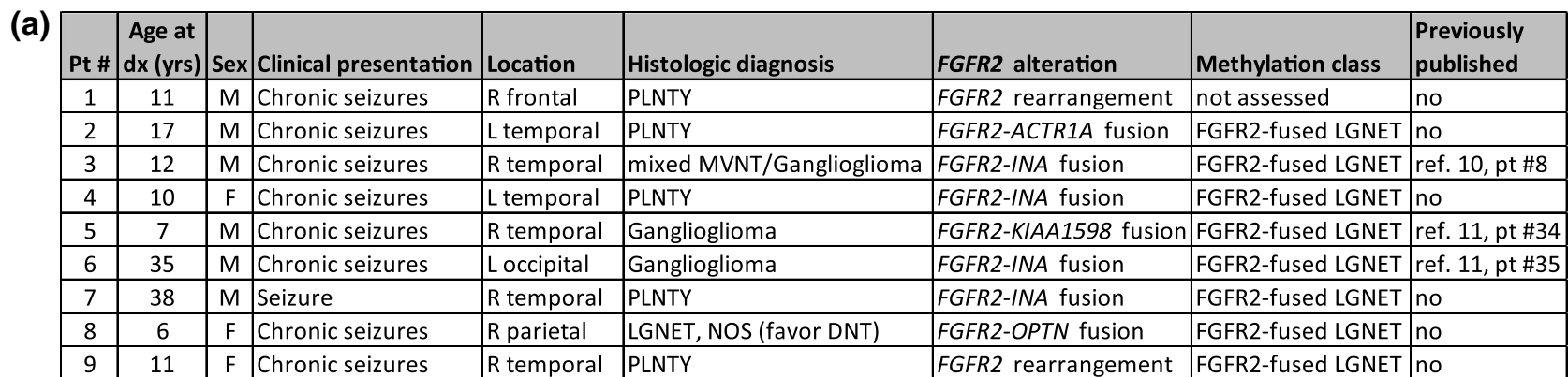
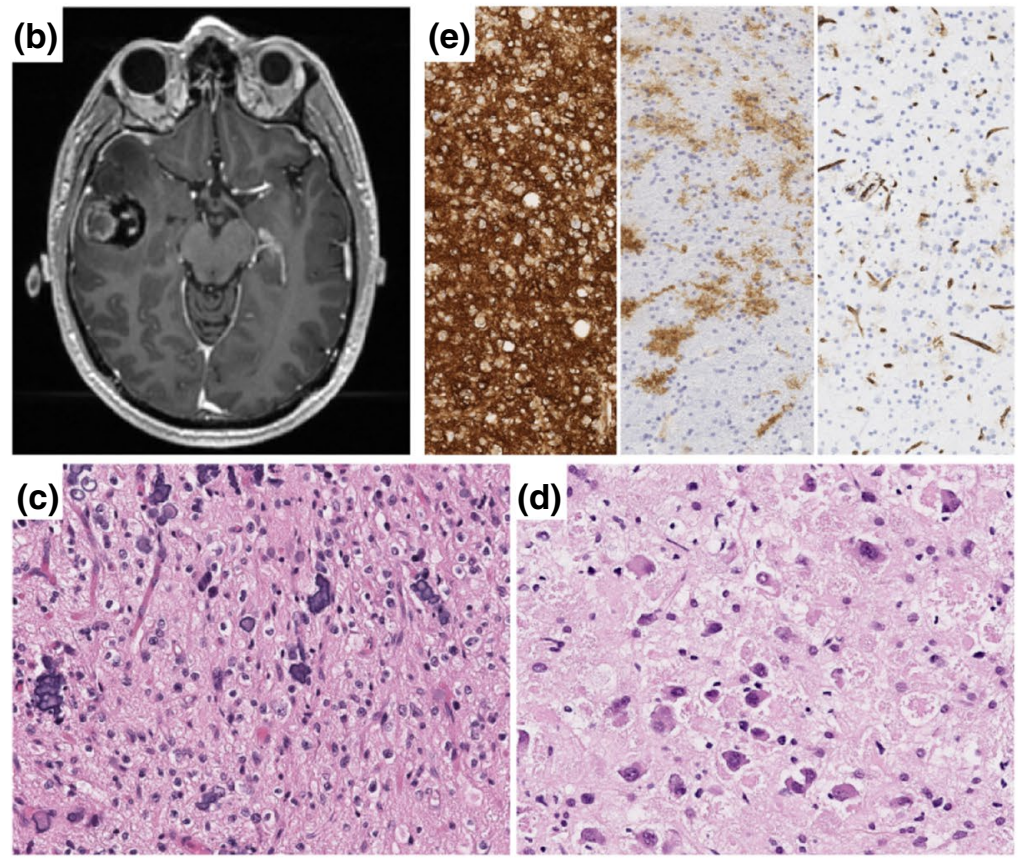

(f)

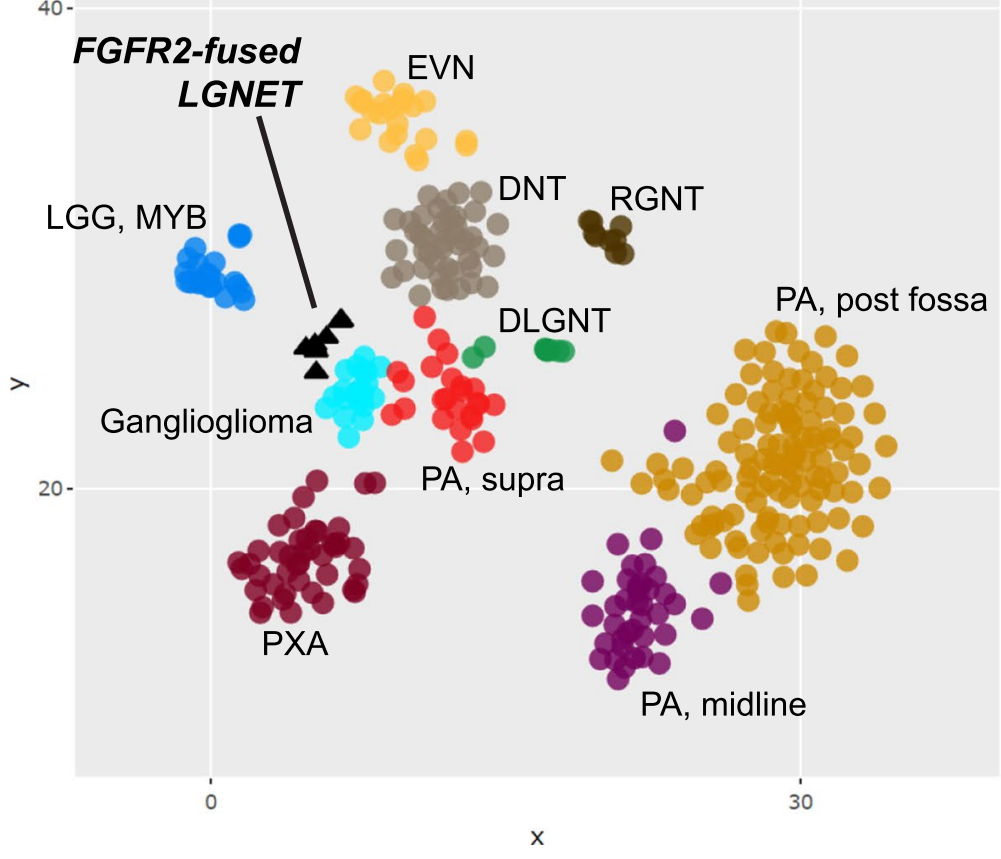

(g)

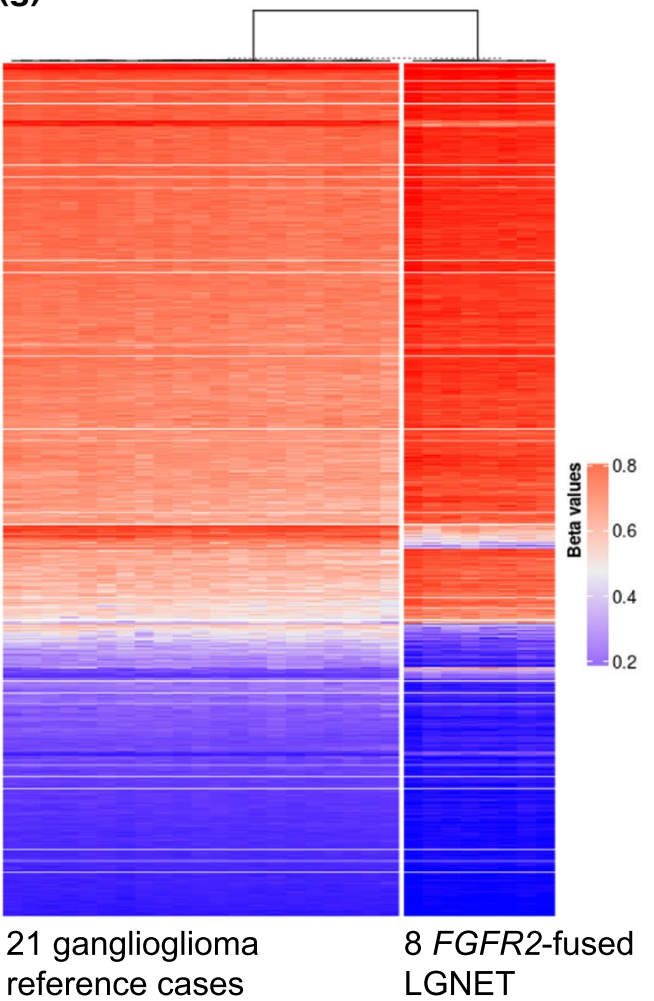

(h)

\begin{tabular}{l}
\hline negative regulation of focal adhesion assembly \\
\hline positive regulation of lamellipodium morphogenesis \\
\hline regulation of establishment of cell polarity \\
\hline retromer complex \\
axonal growth cone \\
\hline retrograde neuronal dense core vesicle transport \\
\hline neuron projection \\
\hline anterograde neuronal dense core vesicle transport \\
\hline positive regulation of amyloid-beta clearance \\
\hline $2.5 \quad 3.0$ \\
\hline \\
\hline
\end{tabular}


४Fig. 1 Clinicopathologic features and epigenomic profiling of lowgrade neuroepithelial tumors harboring $F G F R 2$ gene fusions. a Summary table of the 9 patients with FGFR2-fused LGNET. b MR imaging from patient \#7 demonstrating a solid and cystic lesion within the right temporal lobe of the brain. c Histology from patient \#1 showing characteristic features of PLNTY including round oligodendrocyte-like tumor cells with extensive calcifications. d Histology from patient \#5 showing numerous dysmorphic ganglion cells and eosinophilic granular bodies without calcifications. e Immunochemical staining for CD34 protein showing variable staining patterns in the FGFR2-fused LGNET, including diffuse strong staining of tumor cells (left, patient \#3), abundant ramified cells (middle, patient \#6), and minimal extravascular positivity (right, patient \#8). f tSNE dimensionality reduction plot of genome-wide DNA methylation profiles from $8 F G F R 2$-fused LGNET alongside 346 reference CNS tumors spanning 10 LGNET entities. g Unsupervised hierarchical clustering of DNA methylation data showing segregation of the 8 FGFR2-fused LGNET from a reference cohort of 21 gangliogliomas. h Differential methylation-based Gene Ontology analysis for FGFR2fused LGNET compared to ganglioglioma, represented in a bar plot of $-\log _{10} \mathrm{P}$ values for the most differentially methylated gene networks

profiling on a cohort of 9 patients with LGNET harboring FGFR2 fusions with a diverse range of histologic diagnoses. Three patients had been previously included in our investigations on the genomic landscape of ganglioglioma and MVNT $[10,11]$. The cohort consisted of 6 males and 3 females with median age of 11 years (range 6-38 years), all presenting with chronic seizures (Fig. 1a). Imaging revealed solid and cystic lesions within the cerebral hemispheres (Fig. 1b; Supplementary Fig. 1 [Online Resource 1]). Most patients had gross total resection associated with resolution of seizures and freedom from recurrence during the period of clinical follow-up without adjuvant therapy (Supplementary Table 1 [Online Resource 2]; Supplementary Fig. 2 [Online Resource 1]). Histologically, these were low-grade infiltrative gliomas composed of tumor cells with predominantly round oligodendrocyte-like nuclei (Fig. 1c; Supplementary Fig. 3 [Online Resource 1]; Supplementary Table 2 [Online Resource 2]). Calcifications, often extensive, were present in most but not all tumors. A subset of tumors demonstrated a dysmorphic ganglion cell component and eosinophilic granular bodies (Fig. 1d). Hemosiderin-laden macrophages, indicative of prior intra-tumoral hemorrhage, were also a common finding. One tumor demonstrated vacuolation in both the stroma and ganglion cell component resembling MVNT. Rosenthal fibers, necrosis, and microvascular proliferation were not encountered. Mitotic activity was inconspicuous, and Ki67 labeling was less than $2 \%$ in all examined tumors. Neurofilament and synaptophysin staining revealed entrapped axonal processes in the background of all tumors, and additionally highlighted the dysmorphic ganglion cell component in a subset (Supplementary Table 3 [Online Resource 2]). Immunohistochemistry for CD34 demonstrated diffuse strong labeling of tumor cells characteristic of PLNTY in 6 of 9 tumors, and showed only scattered ramified cells more characteristic of ganglioglioma in the other 3 tumors (Fig. 1e). The original histopathologic diagnosis was PLNTY $(n=5)$, ganglioglioma $(n=2)$, mixed MVNT/ganglioglioma $(n=1)$, and unclassifiable LGNET $(n=1)$. Four tumors demonstrated $F G F R 2$ fusion with $I N A$ as the partner, one tumor each demonstrated fusion with KIAA1598, ACTR1A, and OPTN, and two tumors demonstrated complex FGFR2 rearrangements with uncertain fusion partner based on the targeted DNA sequencing analysis (Supplementary Table 4 [Online Resource 2]). The FGFR2 fusion was the solitary pathogenic alteration identified in all tumors, with an absence of accompanying alterations involving $I D H 1 / 2$, histone $\mathrm{H} 3$ genes, BRAF, NF1, PRKCA, FGFR1, PIK3CA, PIK3R1, PTEN, CDKN2A, TP53, TERT (including promoter region), ATRX, CIC, FUBPI, MYB, and MYBL1 [7]. The quantity of chromosomal copy number aberrations was variable, but no tumors harbored whole arm co-deletion of chromosomes $1 \mathrm{p}$ and $19 \mathrm{q}$, nor were there focal amplifications or homozygous deletions in any tumors (Supplementary Table 5 [Online Resource 2]).

Genome-wide DNA methylation profiling was performed on 8 of the tumors using Infinium EPIC $850 \mathrm{k}$ Beadchips (Illumina) following the manufacturer's recommended protocols (see Supplementary Methods [Online Resource 3]). tSNE clustering of the DNA methylation data alongside reference cohorts of CNS tumors revealed that the FGFR2-fused LGNET formed a single epigenetic group that was distinct from all methylation classes in the current version of the DKFZ classifier v11b4 (Fig. 1f; Supplementary Fig. 4 [Online Resource 1]; Supplementary Tables 6-7 [Online Resource 2]) [2]. Unsupervised hierarchical clustering successfully segregated the 8 FGFR2-fused LGNET from a reference cohort of 21 gangliogliomas (Fig. 1g). Gene Ontology analysis of the most differentially methylated gene regions between FGFR2fused LGNET and ganglioglioma revealed gene networks involved in cell polarity, lamellipodium morphogenesis, and neuronal functions including growth cone projection and core vesicle transport (Fig. 1h; Supplementary Tables 8-10 [Online Resource 2]). tSNE dimensionality reduction of the DNA methylation data alongside 3 histologically-defined cases of PLNTY with FGFR2 fusion from Huse et al. [5] demonstrated close clustering, indicating low-grade glioneuronal tumors with $F G F R 2$ fusion resolve into a single epigenetic group (Supplementary Fig. 5 [Online Resource 1]). Additionally, tSNE dimensionality reduction and unsupervised hierarchical clustering of 3 histologically-defined cases of PLNTY with $B R A F$ p.V600E mutation from Huse et al. [5] demonstrated overall epigenetic similarity to $F G F R 2$-fused LGNET but resolved into a separate methylation subgroup by both 
methodologies, suggesting that PLNTY may be composed of at least two distinct epigenetic subgroups-those with $F G F R 2$ fusion and those with $B R A F$ p.V600E mutation (Supplementary Fig. 5 and 6 [Online Resource 1]).

Here, we demonstrate LGNET with FGFR2 fusions exhibit a spectrum of histologic features, but share an epigenetic signature distinct from all of the reference LGNET methylation classes in the current version $11 \mathrm{~b} 4$ of the DKFZ classifier. Although most FGFR2-fused LGNET belonging to this unique methylation class have histologic features aligning with PLNTY, a subset is devoid of calcifications, have CD34 positivity limited to scattered ramified cells, and contain a prominent ganglion cell component, thereby complicating their differentiation from ganglioglioma based on microscopic features alone. Furthermore, while $B R A F$ and $F G F R I$ alterations are promiscuous and recurrently present across various LGNET types, FGFR2 fusions among LGNET appear to be quite specific to this distinct epigenetic subgroup of glioneuronal tumors. Notably, similar FGFR2 gene fusions are frequent in intrahepatic cholangiocarcinoma for which clinical trials have shown promising efficacy of small molecule tyrosine kinase inhibitors such as erdafitinib [1, 19]. It remains unclear why $F G F R 2$ fusions are selected for in PLNTY and intrahepatic cholangiocarcinoma, whereas FGFR1 alterations are selected for in pilocytic astrocytoma, DNT, RGNT, and EVN, but is likely to reflect differences in response to the ligand-binding specificity for the various fibroblast growth factors [18]. Future studies are required to define the full clinicopathologic spectrum of FGFR2-fused LGNET and the potential efficacy of genomically tailored therapy for affected patients.

Supplementary Information The online version contains supplementary material available at https://doi.org/10.1007/s00401-021-02352-w.

Acknowledgements We thank Jason Huse, Matija Snuderl, David Jones, and Marc Rosenblum for sharing the DNA methylation array data from their previously published PLNTY cohort [5]. C.G.L. is supported by the UCSF Training Program in Translational Brain Tumor Research, National Cancer Institute, National Institutes of Health (T32 CA151022). This study was supported by the NIH Director's Early Independence Award from the Office of the Director, National Institutes of Health (DP5 OD021403) to D.A.S.; a Developmental Research Program Award from the UCSF Brain Tumor SPORE from the National Cancer Institute, National Institutes of Health (P50 CA097257) to D.A.S.; a generous gift from the Morgan Adams Foundation to D.A.S.; a generous gift from the Yuvann Tiwari Foundation to D.A.S.; and the UCSF Glioblastoma Precision Medicine Program sponsored by the Sandler Foundation. We thank the staff of the UCSF Clinical Cancer Genomics Laboratory for assistance with genetic profiling.

Data availability Scanned image files of H\&E and CD34 stained sections from the 9 tumors in this cohort are available for downloading and viewing at the following link: https://figshare.com/projects/Lowgrade_neuroepithelial_tumors_with_FGFR2_fusion/113418. DNA methylation array data files from this study are available from the
Gene Expression Omnibus (GEO) repository under accession number GSE172081 (https://www.ncbi.nlm.nih.gov/geo/). Structural variant and copy number data are available in the electronic supplementary material. Raw sequencing data files are available upon request.

\section{Declarations}

Conflict of interest The authors declare that they have no competing interests related to this report.

Ethical approval This study was approved by the Committee on Human Research of the University of California, San Francisco, with a waiver of patient consent.

Open Access This article is licensed under a Creative Commons Attribution 4.0 International License, which permits use, sharing, adaptation, distribution and reproduction in any medium or format, as long as you give appropriate credit to the original author(s) and the source, provide a link to the Creative Commons licence, and indicate if changes were made. The images or other third party material in this article are included in the article's Creative Commons licence, unless indicated otherwise in a credit line to the material. If material is not included in the article's Creative Commons licence and your intended use is not permitted by statutory regulation or exceeds the permitted use, you will need to obtain permission directly from the copyright holder. To view a copy of this licence, visit http://creativecommons.org/licenses/by/4.0/.

\section{References}

1. Arai Y, Totoki Y, Hosoda F et al (2014) Fibroblast growth factor receptor 2 tyrosine kinase fusions define a unique molecular subtype of cholangiocarcinoma. Hepatology 59:1427-1434

2. Capper D, Jones DTW, Sill M et al (2018) DNA methylationbased classification of central nervous system tumours. Nature 555:469-474

3. Deng MY, Sill M, Chiang J et al (2018) Molecularly defined diffuse leptomeningeal glioneuronal tumor (DLGNT) comprises two subgroups with distinct clinical and genetic features. Acta Neuropathol 136:239-253

4. Hou Y, Pinheiro J, Sahm F et al (2019) Papillary glioneuronal tumor (PGNT) exhibits a characteristic methylation profile and fusions involving PRKCA. Acta Neuropathol 137:837-846

5. Huse JT, Snuderl M, Jones DT et al (2017) Polymorphous lowgrade neuroepithelial tumor of the young (PLNTY): an epileptogenic neoplasm with oligodendroglioma-like components, aberrant CD34 expression, and genetic alterations involving the MAP kinase pathway. Acta Neuropathol 133:417-429

6. Jain P, Surrey LF, Straka J et al (2018) Novel FGFR2-INA fusion identified in two low-grade mixed neuronal-glial tumors drives oncogenesis via MAPK and PI3K/mTOR pathway activation. Acta Neuropathol 136:167-169

7. Kline CN, Joseph NM, Grenert JP et al (2017) Targeted nextgeneration sequencing of pediatric neuro-oncology patients improves diagnosis, identifies pathogenic germline mutations, and directs targeted therapy. Neuro Oncol 19:699-709

8. Lucas CG, Gupta R, Doo P et al (2020) Comprehensive analysis of diverse low-grade neuroepithelial tumors with FGFR1 alterations reveals a distinct molecular signature of rosette-forming glioneuronal tumor. Acta Neuropathol Commun 8:151

9. Lucas CG, Villanueva-Meyer JE, Whipple N et al (2020) Myxoid glioneuronal tumor, PDGFRA p. K385-mutant: 
clinical, radiologic, and histopathologic features. Brain Pathol 30:479-494

10. Pekmezci M, Stevers M, Phillips JJ et al (2018) Multinodular and vacuolating neuronal tumor of the cerebrum is a clonal neoplasm defined by genetic alterations that activate the MAP kinase signaling pathway. Acta Neuropathol 135:485-488

11. Pekmezci M, Villanueva-Meyer JE, Goode B et al (2018) The genetic landscape of ganglioglioma. Acta Neuropathol Commun 6:47

12. Qaddoumi I, Orisme W, Wen J et al (2016) Genetic alterations in uncommon low-grade neuroepithelial tumors: BRAF, FGFR1, and MYB mutations occur at high frequency and align with morphology. Acta Neuropathol 131:833-845

13. Rivera B, Gayden T, Carrot-Zhang J et al (2016) Germline and somatic FGFR1 abnormalities in dysembryoplastic neuroepithelial tumors. Acta Neuropathol 131:847-863

14. Ryall S, Zapotocky M, Fukuoka K et al (2020) Integrated molecular and clinical analysis of 1,000 pediatric low-grade gliomas. Cancer Cell 37:569-583

15. Sievers P, Appay R, Schrimpf D et al (2019) Rosette-forming glioneuronal tumors share a distinct DNA methylation profile and mutations in FGFR1, with recurrent co-mutation of PIK3CA and NF1. Acta Neuropathol 138:497-504
16. Sievers P, Schrimpf D, Stichel D et al (2020) Posterior fossa pilocytic astrocytomas with oligodendroglial features show frequent FGFR1 activation via fusion or mutation. Acta Neuropathol 139:403-406

17. Sievers P, Stichel D, Schrimpf D et al (2018) FGFR1:TACC1 fusion is a frequent event in molecularly defined extraventricular neurocytoma. Acta Neuropathol 136:293-302

18. Turner N, Grose R (2010) Fibroblast growth factor signaling: from development to cancer. Nat Rev Cancer 10:116-129

19. Wu YM, Su F, Kalyana-Sundaram S et al (2013) Identification of targetable FGFR gene fusions in diverse cancers. Cancer Discov 3:636-647

20. Zhang J, Wu G, Miller CP et al (2013) Whole-genome sequencing identifies genetic alterations in pediatric low-grade gliomas. Nat Genet 45:602-612

Publisher's Note Springer Nature remains neutral with regard to jurisdictional claims in published maps and institutional affiliations. 